\title{
Unequal clustering algorithm with IDA* multi-hop routing to prevent hot spot problem in WSNs
}

\author{
Ahmed A. Alkadhmawee ${ }^{1}$, Mohammed A.Altaha ${ }^{2}$, Wisam Mahmood Lafta ${ }^{3}$ \\ ${ }^{1}$ College of Education for Human Sciences, Universitiy of Basrah, Iraq \\ ${ }^{2}$ College of Veterinary, Universitiy of Basrah, Iraq \\ ${ }^{3}$ Department of Computer Science, Universitiy of Technology, Iraq
}

\begin{tabular}{l}
\hline Article Info \\
\hline Article history: \\
Received Jan 4, 2020 \\
Revised Mar 6, 2020 \\
Accepted Mar 27, 2020 \\
\hline
\end{tabular}

\section{Keywords:}

Hot spot problem

IDA* algorithm

Unequal clustering algorithm

Wireless sensor networks

\begin{abstract}
Energy conservation techniques are considered as the primary means of lengthening the lifetime of Wireless Sensor Networks (WSNs). Clustering is a useful technique that achieves long-term operation of the network. Cluster heads that are near the base station are expected to die early because they are responsible for collecting data that comes from far away in addition to their own data, thereby leading to problem of hot spot in the WSN. This paper presents a new protocol that uses an unequal clustering algorithm with an IDA* routing method to address the hot spot problem. The base station divides the network into three levels of unequal sizes of clusters. The base station takes into consideration the energy level and the distance from the base station for cluster-head selection in each cluster. The cluster head will be changed based on the energy threshold for each cluster. The proposed method uses an IDA* algorithm for efficient multi-hop routing in the network. The uneven clustering algorithm reduces the energy consumption of the nodes, thereby minimising the hot spot problem. The obtained simulation results prove that our approach increases the load balancing, improves the stability and prolongs the network lifetime compared with other related approaches.
\end{abstract}

Copyright $@ 2020$ Institute of Advanced Engineering and Science. All rights reserved.

\section{Corresponding Author:}

Ahmed A. Alkadhmawee,

Department of Computer Enginerring,

Universitiy of Basrah, Iraq.

Email: ahmedadel1949@gmail.com

\section{INTRODUCTION}

Wireless Sensor Networks (WSNs) offer a great combination of distributed sensing, regulator communication and computation ability; this means that WSNs lend themselves to countless applications. Fundamentally, WSNs have enabled a paradigm shift in both civilian and military monitoring fields and have formed a basis for covering a wide range of applications related to medical, environmental and industrial control as well as security. WSNs consist of an array of wireless sensors that are interconnected by a communication network. These sensor nodes can periodically send reports of their sensed data to neighbouring nodes or to the base station, which is able to connect with the internet or to existing communication infrastructure [1].

In WSNs, the battery's sensor nodes operate with a limited power supply, and it is not practical to replace or recharge them. The limited energy resources have a significant influence on prolonging the WSN lifetime [2-5]. However, It is necessary to implement certain techniques or mechanisms in order to deal with those constraints.

Clustering is a useful technique that achieves long-term operation of the network and can help to solve some of the node's constraints [6-8]. Clustering has been adopted to increase the efficiency of the WSN by organising the sensor nodes into clusters. The sensor nodes for each cluster have a cluster head. The data 
routing inside the cluster is known as intra-cluster data routing while the data routing between the cluster heads is known as inter-cluster data routing.

Many clustering protocols use a multi-hop of cluster heads while transmitting the aggregation data to the base station in order to minimise the energy consumption of the nodes in the network. In multi-hop clustering, the cluster heads nearest to the base station act as transmitting stations for other cluster heads in addition to aggregating the data of their own clusters. The cluster heads nearest the base station have the largest traffic load and deplete their energy quicker than other nodes. Therefore, these cluster heads expected to die early, leading to hot spot problem in the WSN [9-13].

Recently, many studies have been proposed to analyze existing clustering techniques. Hienzelman et al. [14] proposed LEACH clustering protocol. LEACH selects cluster heads based on probability by generated a random number between 0 and 1 for each node. Nodes that have a number less than the threshold value elect themselves as cluster heads. There are several major drawbacks to LEACH: the size of the clusters is uneven for different rounds, the cluster heads transmitted the information directly to the base station without any relay nodes and the protocol does not take the nodes' remaining energy into consideration when selecting the cluster heads. Coyle et al. [15] have presented a layered approach-based clustering protocol called the EEHC algorithm. This algorithm segments the network into several layers. In the lower layer, the cluster head receives inforamtion and transmits it is to the base station by the upper layer cluster heads. Data delivery delay is the main obstacle of this algorithm. Liu et al. have presented the LPGCRA protocol to maximise the network lifetime [16]. This protocol considers only the energy level when selecting the cluster head. The cluster heads transmit the information directly to the base station. Hence, more energy is wasted. The Leach-A* algorithm has been proposed by $\mathrm{H}$. Chen et al. [17] to extend the WSN lifetime. The Leach algorithm performs the process of selection cluster head while the $A^{*}$ algorithm uses for inter-cluster communication. Leach algorithm neglects the energy level during the cluster head selection process.

The major disadvantage of all of the all above protocols is the problem of hot spot, which decreases the network lifetime. The cluster heads nearest the base station have the largest traffic load and deplete their energy quicker than other nodes. Many researches proposed methods on unequal cluster formation to address the problem of hot spot.Li et al. [18] proposed Energy Efficient Unequal Clustering (EEUC). EEUC reduces the problem of hot spot by using the approach of uneven cluster configuration and a multi-hop routing method. The clustering algorithm partitions the network into an uneven cluster. The clusters nearest to the base station are smaller than other clusters. The cluster head selects a relay node corresponding to the remaining energy of the node and its distance from the base station. EEUC depends on the residual energy of the node for selection cluster head. The main obstacle of this protocol is the probabilistic way of selecting a node as a cluster head. Bagci and Yazici [19] presented an energy-aware fuzzy unequal clustering algorithm. The approach depends on fuzzy logic, which uses the distance to the base station and the energy level as parameters for selecting the cluster head in the network. However, the approach does not take into account the main factor during performance evaluation, which is the energy that is exhausted during inter-cluster communication. Lee et al. [20] introduced a new protocol called LUCA. This scheme keeps the small clusters nearest to the base station and their cluster heads focused on inter-cluster communication, while the cluster heads in larger clusters focus on intra-cluster communication. Cluster size is not optimal and hence the energy consumption of the network is increased.Unequal clustering protocol called EBUC has been proposed by Jiang et al. [21]. It bases itself on the PSO algorithm to select cluster heads and to form uneven clusters. EBUC uses a greedy method for inter-cluster routing. The greedy algorithm depends on the remaining energy and distance to the base station to select a relay node. Although the network lifetime is improved with a reduced rate of dead nodes, the hot spot problem is still the main obstacle of this protocol.

This paper presents an unequal clustering method that divides the network into unequal sizes of levels in which each level has a number of clusters. Smaller clusters are placed closer to the base station, and the size of the cluster increases the farther away it is from the base station. The energy consumption of the cluster heads that are nearer the base station is reduced due to the short distance for the transmission of data, thereby minimising the hot spot problem. However, the communication cost is higher for the clusters that are located farther away from the base station because of the larger size of these clusters. The proposed method uses multi-hop intra-cluster communication to solve this problem. The IDA* routing method is utilised to find the optimum path for intra-cluster communication. Furthermore, our method uses the IDA* algorithm for inter-cluster communication to complete the routing path from the cluster heads to the base station.

Our approach takes into consideration the highest energy level and the minimum distance to the base station during cluster-head selection. The cluster head will be changed based on the energy threshold for each cluster. The energy threshold makes the cluster heads play their role for multiple rounds without changing. In the remainder of this paper, Section 2 presents the IDA* algorithm. The proposed system model for this 
work describes in Section 3. Section 4 presents the proposed method which includes the clustering formation phase, routing phase and data collection phase. In Section 5, the performance evaluation and analysis are explained. Concludes this paper in Section 6.

\section{ITERATIVE DEEPENING A-STAR (IDA*) ALGORITHM}

Korf proposes the IDA* algorithm to find the optimal solutions for typical problems such as the cutting stock problem, floorplan optimisation and the traveling salesman problem [22]. The IDA* algorithm utilises iterative deepening for the efficient usage of memory space and uses the $A^{*}$ search algorithm for an effective usage of heuristic knowledge [23]. The IDA* algorithm uses the evolution function (f[s]) to find the route path from the start node to the goal node. This function can be represented by (1) where $h(s)$ refers to the distance from the node $s$ to the destintion node and $g(s)$ refers to the cost path to reach from node $s$ to the goal node:

$$
\mathrm{f}(\mathrm{s})=g(s)+h(s)
$$

The IDA* algorithm depends on cut-off value. The cut-off value is used to select the nodes that should be expanded along the current path. The nodes will be expanded when their f-value is larger than the cut-off. The cut-off value is recomputed iteratively by taking the smallest f-value that exceeded the previous search [24]. The IDA* algorithm ends when the goal is reached and stops when no more nodes are found to expand.

\section{SYSTEM MODEL}

\subsection{Network model}

The sensing area consists of a set of sensor nodes that are distributed randomly over an $\mathrm{M} * \mathrm{M}$ square area. In this paper, the following assumptions will be a consideration regarding the network model and sensor nodes:

a) There is a static base station that has sufficient resources to manage the network and collect the data.

b) The base station becomes aware of all the nodes' records in the network like location, node identification and residual energy.

c) Every node in the network is static, unaware of their location and has a unique identification.

d) Initially, all sensors have the same amount of energy.

e) The sensor nodes have the same computation and communication capabilities.

\subsection{Energy model}

In a WSN, sensor nodes consume most of their energy while transmitting and receiving data. The first radio model proposed in [25] is adopted to calculate the energy consumption. This model computes the energy that is dissipated when transmitting or receiving a message of $\mathrm{m}$ bits between two nodes $(s, n)$ over a distance $\mathrm{d}$. The total energy consumption for node $\mathrm{s}$ can be calculated as follows:

$$
\begin{aligned}
& E_{c}(s, n)=E_{c_{T x}}(s, n)+E_{c_{R x}}(s, n) \\
& E_{c}(i, j)=m\left(E_{a m p} \times d^{2}+E_{\text {elec }}\right)+m \times E_{\text {elec }} \\
& E_{c_{T x}}(s, n) \text { and } E_{c_{R x}}(s, n) \text { are the energy dissipated for transmitting and receiving, }
\end{aligned}
$$
respectively. $E_{\text {elec }}$ is the amount of dissipated energy for a transmitter or receiver, and $E_{a m p}$ is the energy usage for the transmit amplifier. $E_{\text {elec }}$ is based on many factors like filtering, modulation, the digital coding and the spreading of the signal, while $\mathrm{E}_{\mathrm{amp}}$ is based on the acceptable bit rate error and the distance between the transmitter and receiver.

\section{PROPOSED METHOD}

\subsection{Cluster configuration phase and cluster-head selection}

The cluster configuration is performed only once at the base station to eliminate the clustering formation overhead in each round. The base station divides the sensing area into three rectangular levels of unequal size, and each level is subdivided to the number of clusters. The levels are the front level, medium level and rear level. The front level denotes the first level that is nearest to the base station. The medium level is beyond the front level, while the rear level indicates the level that is the farthest away

Unequal clustering algorithm with IDA* multi-hop routing to prevent hot spot... (Ahmed A. Alkadhmawee) 
from the base station. The size of the front level depends on the threshold distance in the first radio model [20]. The sensor nodes within the threshold distance and nearest to the base station are considered as the front level sensor nodes. The sensor nodes at the front level can connect directly to the base station. The medium and rear levels are two and three times the size of the front level, respectively. The objective of designing levels in this way is to reduce the number of cluster heads in each round and prevent the hot spot problem.

According to [9], the optimum number of clusters has an inverse co-relation with an increased distance to the base station. This indicates that level $\mathrm{L}$ should have a higher number of clusters than level L-1. In our proposed method, the number of clusters in the front, medium and rear levels is $n, n-1$ and n-2, respectively. Figure 1 illustrates the network partition and cluster configuration process.

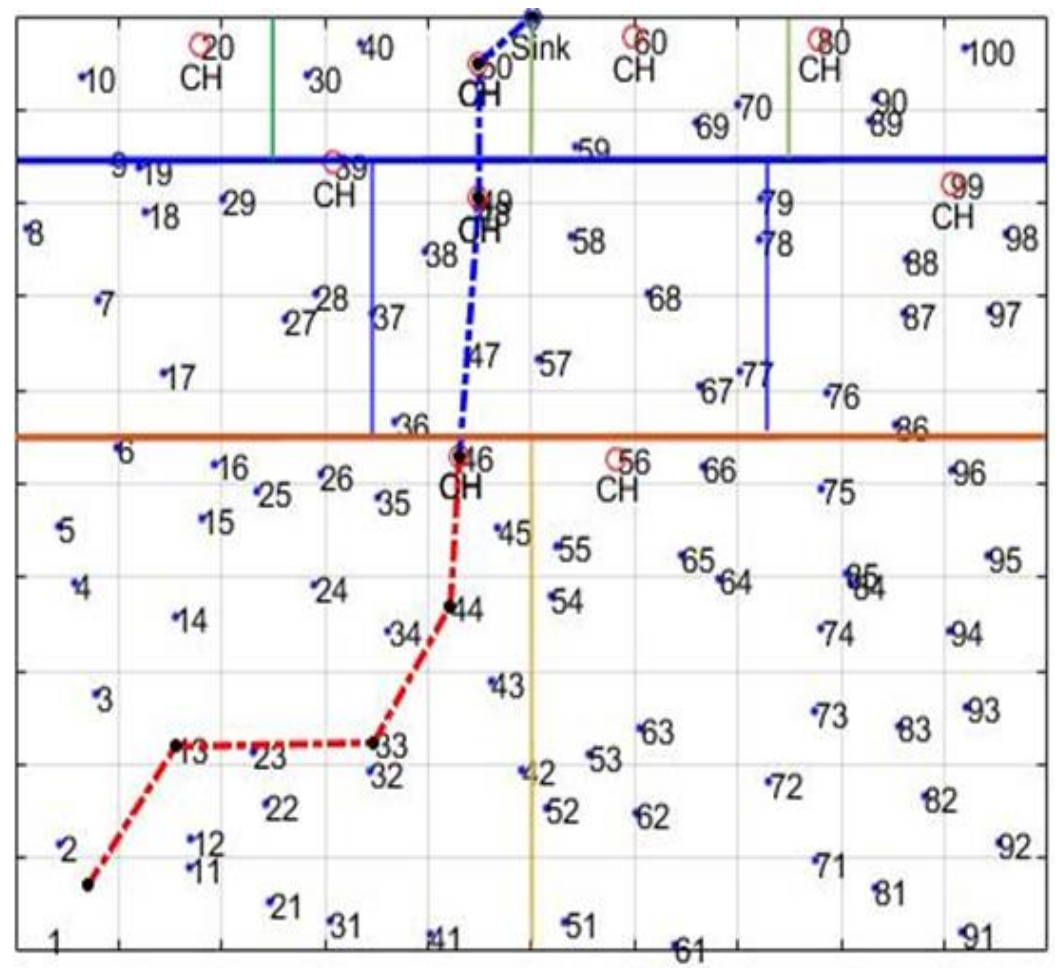

Figure 1. The network partition and clusters configuration process

The cluster heads will be assigned by the base station before starting the first round. The base station puts the information of each node inside the selection table, which is utilized to find the best cluster head. The selection table includes some of the information for each node in the cluster, like the node identification, residual energy, distance to the base station and hop level. The selection table for the base station is always updated at the beginning of every round. The sensor nodes for each cluster rank in the selection table based on their energy level and their location from the base station. The node that has the highest residual energy and is nearest to the base station will be selected as the cluster head according to the following formulas:

$$
\begin{aligned}
& \operatorname{Node}_{\text {Weight }}(i)=\left(R_{\text {res }}(i), \operatorname{Dist}_{\text {toBS }}(i)\right) \\
& \text { Clusterhead }_{\text {selection }}=\left(\operatorname{Max}\left(R_{\text {res }}\right), \operatorname{Min}\left(\text { Dist }_{\text {toBS }}\right)\right.
\end{aligned}
$$

where $R_{\text {res }}(i)$ is the residual energy of node $\mathrm{i}, \operatorname{Dist}_{t o B S}(i)$ is the distance of node $\mathrm{i}$ to the base station. $\operatorname{Dist}_{\text {toBs }}(i)$ is determined by the Euclidian distance.

The base station checks the residual energy of cluster heads after each round. Only cluster heads with an energy level above the energy threshold continue their job. The energy threshold ( $\left.E_{\text {threshold }}\right)$ can be determined for each cluster by the (6), where $\mathrm{N}$ is the number of nodes in the network and $\alpha$ is a constant value. The energy threshold makes the cluster heads play their role for multiple rounds without changing, thereby reducing the energy that would be wasted if a new cluster head was selected in each round. 


$$
E_{\text {threshold }}=\alpha \times \frac{\sum_{i=1}^{N} R_{\text {res }}(i)}{N}
$$

Cluster heads transmit a message at the beginning of every round to their nodes. The message contains the cluster head identification (id) and hop level $=0$. The neighbouring nodes of the cluster head update their hop level and forward the message to the next hop, incrementing the hop level by 1 . The hop level value for the front, medium and rear clusters does not exceed 0,1 and 2, respectively.

\subsection{Routing phase}

In the proposed method, the IDA* routing algorithm will be used for intra-cluster and inter-cluster communication processes. In intra-cluster routing, the nodes of the front clusters send data to their cluster head directly. The nodes of the medium and rear clusters support multi-hop communication. The nodes of the medium and rear clusters utilize the IDA* method to select their route path. The IDA* algorithm uses the energy level and the distance to the base station to select the routing path. This algorithm applied the following evaluation function (f[n]) to each node $\mathrm{n}$ of the network:

$$
f(n)=R_{\text {res }}(n)+\frac{1}{h(n)}
$$

Where $R_{\text {res }}(n)$ denotes the residual energy of node $\mathrm{n}$ and $h(n)$ denotes the distance from node $\mathrm{n}$ to the base station. The IDA* algorithm uses the cut-off value to select the number of nodes that should be expanded. The cut-off value is recomputed iteratively by taking the minimum f-value among all nodes that expanded in the previous search. The cut-off value can be expressed as follows:

$$
\text { cutoff }=\operatorname{Min}_{\text {previously }}(f(n))
$$

Initially, the cut-off takes the f-value of the start node. $\mathrm{N}$ nodes will be expanded only when their $\mathrm{f}(\mathrm{n})$ is larger than the cut-off value. The start node sends data to the neighbouring node that has the largest f-value and has exceeded the cut-off. In the next hop, the cut-off value is recalculated by (8). N nodes that have (f[n] > cut-off) should be expanded. The node that has the largest f-value among the expanded nodes will be selected in the next hop. This process is repeated until the route is completed the cluster head.

In inter-cluster routing, cluster head transmit the aggregated data to the upper level by using the IDA* algorithm. The IDA* algorithm calculates the cut-off value at each level by taking the minimum f-value among all cluster heads that expanded from the previous level. The IDA* algorithm compares the f-value of each cluster head with the cut-off. If the f-value of the cluster head is larger than the cut-off, then the cluster head is expanded. One expanded cluster head will be chosen as the best relay; this cluster head has the largest $\mathrm{f}$-value. This process is repeated until the route is completed to the base station.

\subsection{Data collection phase}

In this phase, we have formed the tree structure for data collection in each cluster to reduce the work of the cluster head. The cluster head acts as a root of the tree and there are two or three levels of member nodes in medium and rear clusters. The load of aggregation is distributed between the member nodes in these different sizes of clusters. As a result, the cluster head spends less energy.

The base station uses a TDMA schedule to allocate the number of slots for cluster members. The number of allocated slots could be different for each cluster according to the number of nodes. Each node is assigned a unique TDMA. When the cluster head sends its id and hop level, the sensor nodes then know their TDMA and can set their hop level. Initially, the sensor node that has the data checks the hop level of neighbouring nodes. When the cluster head is out of range, the sensor node transmits sensed data to the neighbouring node that has a lower hop level in their TDMA. The sensor node go to a sleep state after it has finished transmitting all the data with negligible power consumption. The intermediate node forwards these aggregation packets to the cluster head and then goes into a sleep state. The cluster head transmits the packets to the base station through upper level cluster heads. The process is repeated until all rounds end. The flowchart for the data collection procedure is illustrated in Figure 2. 


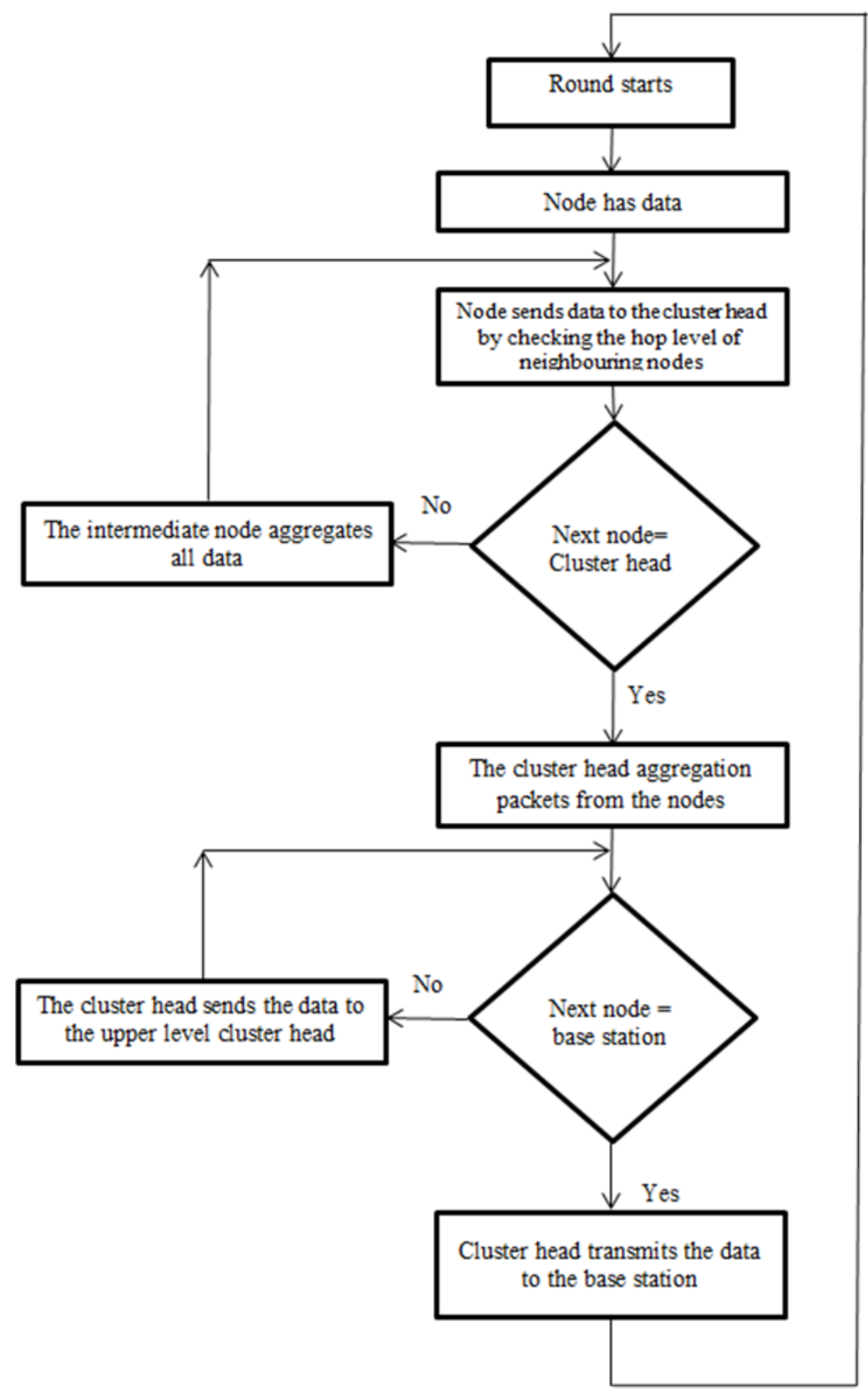

Figure 2. The flowchart for the data collection phase

\section{RESULTS OF THE PROPOSED METHOD}

\subsection{Comparative methods}

The proposed method was compared with related methods. The first approach (EEUC) used an uneven clustering method and multi-hop routing to minimise the hot spot problem [13]. The second method for routing comparison used the IDA* algorithm to find inter-cluster and intra-cluster communications and depended on parameters like the distance to the base station and energy level.

\subsection{Simulation parameters}

A MATLAB programming environment was used to simulate the proposed clustering approach. The simulation was performed in an environment of $100 \times 100 \mathrm{~m}^{2}$ where 100 sensor nodes are spread randomly and the base station is located at $100 \times 50$. Every node has a fixed radio range up to $35 \mathrm{~m}$ with an initial energy of $0.5 \mathrm{j}$. The list of all parameters for the simulation environment is exhibited in Table 1. 
Table 1. Simulation parameters

\begin{tabular}{cc}
\hline Parameter & Value \\
\hline Network area & 10 \\
Number of nodes & 100 \\
Transmission radio range & $35 \mathrm{~m}$ \\
Initial energy & $0.5 \mathrm{j}$ \\
Base station location & $100 \times 50 \mathrm{~m}^{2}$ \\
Amplifier transmitting energy $\left(\mathrm{E}_{\mathrm{amp}}\right)$ & $100 \mathrm{~nJ} / \mathrm{bit} / \mathrm{m}^{2}$ \\
Electronics energy ( $\left.\mathrm{E}_{\mathrm{elec}}\right)$ & $50 \mathrm{~nJ} / \mathrm{bit}$ \\
Size of Data Packet & $2 \mathrm{k} \mathrm{bit}$ \\
\hline
\end{tabular}

\subsection{Performance evaluation}

The purpose of this research was to increase the lifetime of the network by balancing energy consumption and reducing the hot spot problem. To achieve this purpose, the performance of the proposed work was evaluated. The count of nodes still alive, the death of the first node, the average energy of the network and the message delay were determined. The simulation results of our method have compared with EEUC and IDA* protocols.

The number of nodes still alive is an essential metric by researchers to evaluate the network lifetime. The count of nodes still alive after a certain round is displayed in Figure 3. It can be seen from this figure that the proposed work has the largest number of nodes still alive compared with other studies. The routing criteria and clustering method proposed by our protocol are the main reasons for the increased number of nodes still alive in the network.

The death of the first node is the key factor for measuring the performance of the network stability. The number of rounds until the death of the first node in the network represents the stability period. The simulation results of the network stability for the protocols is determined in Table 2. In Table 2, the first node died in (IDA* EEUC) at round 2001 and 1333, respectively, whereas our protocol performed round 2802 before the first node died. As a result, the presented protocol is more efficient in terms of network stability compared with other protocols. The uneven size of clusters and the optimised cluster head rotation are the major contributors to reducing the hot spot problem and increasing node lifetime near the base station. As well, these factors evenly balanced the energy consumption between the nodes, thereby increasing the network lifetime.

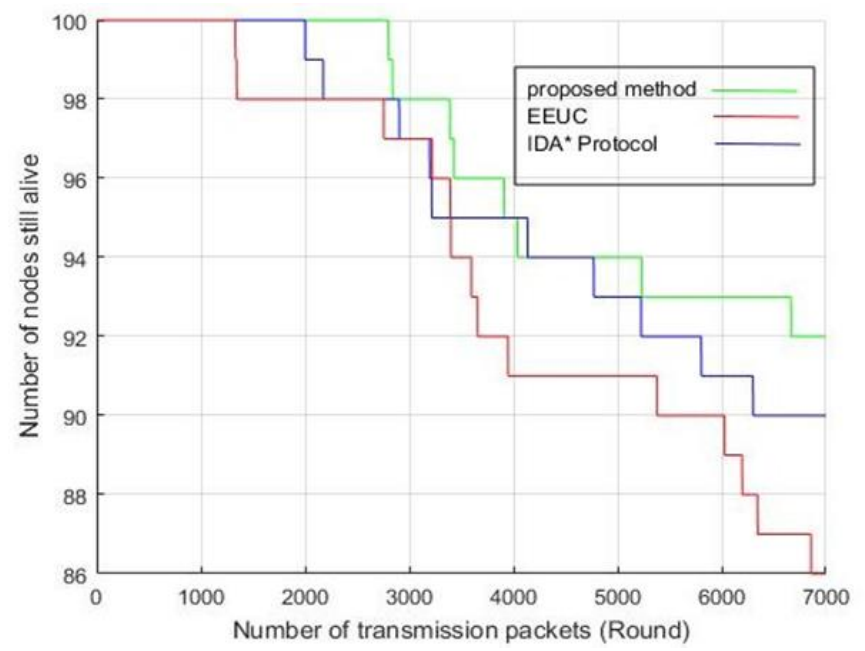

Table 2. Simulation results of the network stability

\begin{tabular}{cc}
\multicolumn{2}{c}{ network stability } \\
\hline Protocol & $\begin{array}{c}\text { The first node died } \\
\text { at round }\end{array}$ \\
\hline The proposed method & 2802 \\
IDA* protocol & 2001 \\
EEUC protocol & 1333 \\
\hline
\end{tabular}

Figure 3. Nodes still alive with respect to round numbers

The simulation result of the average residual energy for all protocols is shown in Figure 4. This figure illustrates that the residual energy of our network is more than (EEUC) and (IDA*) protocols. The proposed protocol reduced energy consumption by using the IDA* algorithm for intra-cluster routing. In addition, the presented protocol distributed the energy consumption between nodes by selecting the appropriate cluster head. Consequently, the load balancing was increased, and the average energy of the network was improved. 
The time of the data packet transmissions is a significant parameter for many applications relying on WSNs. The transmission delay for the three approaches after a number of rounds is shown in Figure 5. The proposed approach clearly has less of a transmission delay than other approaches. This is because of the nature of the IDA* algorithm, which expanded only the nodes that exceeded the cut-off value instead of expanding all nodes in the path. The proposed protocol can be used in information access for emergency rescue, monitoring and control.

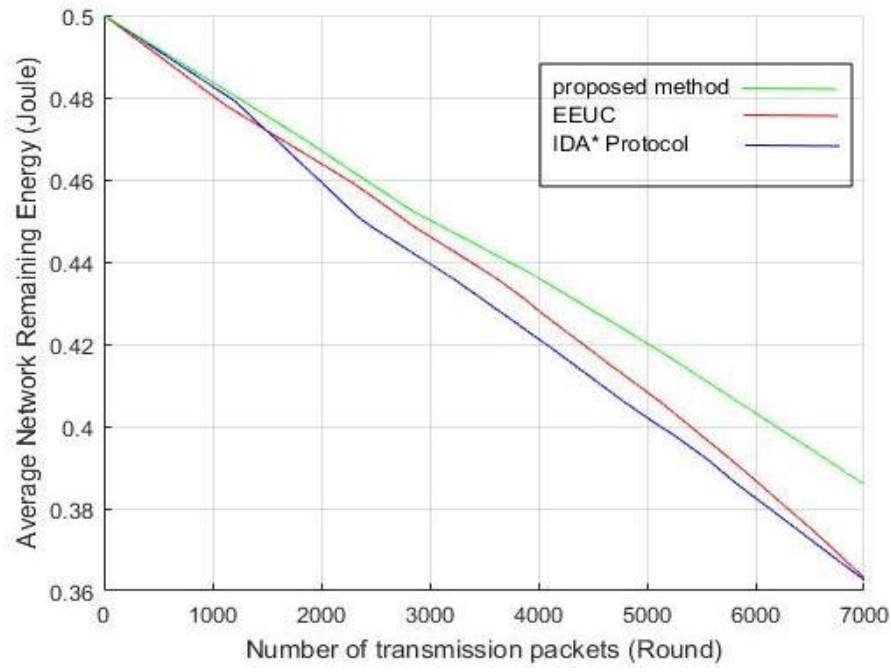

Figure 4. Average residual energy for all protocols

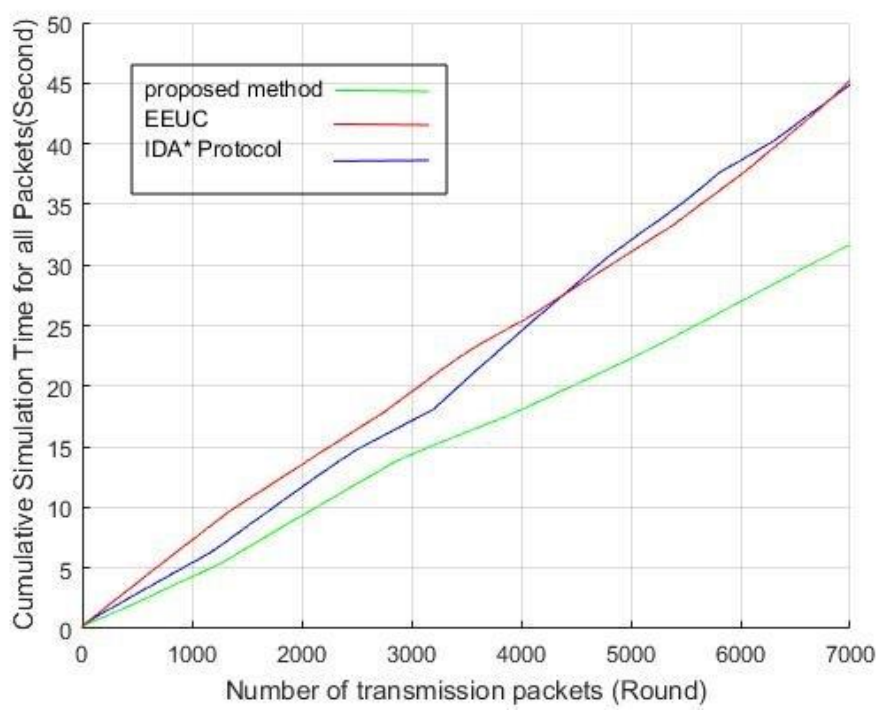

Figure 5. The transmission delay for all protocols

\section{CONCLUSION}

This paper introduced a new unequal clustering mechanism for the WSN that is built on area levelling: front, medium and rear levels. Moreover, this paper presented a new algorithm for data routing through network levels: the IDA* algorithm. The proposed clustering helps to reduce the extra burden of nodes in front clusters, thus addressing the hot spot problem. The IDA* multi-hop method reduces the message overhead as well as the number of clusters in medium and rear clusters. Experiments were conducted to evaluate the proposed protocol. The results show that our approach has good performance in terms of improved energy efficiency, enhanced network stability and extended network lifetime. 


\section{REFERENCES}

[1] A. M. Krishnan and P. G. Kumar, "An effective clustering approach with data aggregation using multiple mobile sinks for heterogeneous wsn,” Wireless Personal Communications, vol. 90, no. 2, pp. 1-12, 2015.

[2] N. A. Pantazis, et al., "Energy-Efficient Routing Protocols in Wireless Sensor Networks: A Survey," in IEEE Communication Survey \& Tutorials, vol. 15, no. 2, pp. 551-591, 2013.

[3] N. A. Pantazis and D. D. Vergados, "A survey on power control issues in wireless sensor networks," IEEE Communications Surveys and Tutorials, vol. 9, no. 4, pp. 86-107, 2007.

[4] G. N. Basavaraj and C. D. Jaidhar, "Low latency and energy efficient cluster based routing design for wireless sensor network," Indonesian Journal of Electrical Engineering and Computer Science, vol. 13, no. 2, pp. 615-625, Feb 2019.

[5] Z. Dzulkurnain, et al., "Internet of things (IoT) based traffic management \& routing solution for parking space," Indonesian Journal of Electrical Engineering and Computer Science (IJEECS), vol. 15, no. 1, pp. 336-345, 2019.

[6] S. Shivapur, et al., "Load balancing techniques in wireless sensor networks: a comparative study," International Journal of Emerging Technology in Computer Science and Electronics, vol. 14, no. 2, pp. 218-223, 2015.

[7] P. Nayak and A. Devulapalli, "A Fuzzy Logic-Based Clustering Algorithm for WSN to Extend the Network Lifetime,” IEEE Sensors Journal, vol. 16, no. 1, pp. 137-144, 2016.

[8] D. Jia, et al., "Dynamic Cluster Head Selection Method for Wireless Sensor Network," IEEE Sensors Journal, vol. 16, no. 8, pp. 2746-2754, 2015.

[9] S. Kurt and B. Tavli, "Path-Loss Modeling for Wireless Sensor Networks: A review of models and comparative evaluations," IEEE Antennas and Propagation Magazine, vol. 59, no. 1, pp. 18-37, 2017.

[10] H. Asharioun, et al., "A survey on analytical modeling and mitigation techniques for the energy hole problem in corona-based wireless sensor network," Wireless Personal Communications, vol. 81, no. 1, pp 161-187, 2015.

[11] M. T. Ruiz, et al., "Innovative services and applications of wireless sensor networks: Research challenges and opportunities," International Journal of Distributed Sensor Networks, vol. 14, no. 5, pp. 1-4, 2018.

[12] I. F. Akyildiz, et al., "Wireless sensor networks: a survey," Computer networks, vol. 38, no. 4, pp. 393-422, 2002.

[13] W. Zhang, et al., "An Energy-Efficient Ring Cross-Layer Optimization Algorithm for Wireless Sensor Networks," IEEE Access, vol. 6, pp. 16588-16598, 2018.

[14] W. R. Heinzelman, et al., "Energy-efficient communication protocol for wireless microsensor networks," in Proceedings of the 33rd annual Hawaii international conference on System sciences, vol. 2, pp. 1-10, 2000.

[15] S. Bandyopadhyay and E. J. Coyle, "An energy efficient hierarchical clustering algorithm for wireless sensor networks," Twenty-Second Annual Joint Conference of the IEEE Computer and Communications Societies, vol. 3, pp. 1713-1723, 2003.

[16] W. D. Liu, et al., "A low power grid-based cluster routing algorithm of wireless sensor networks," in Proceedings of the 2010 International Forum on Information technology and applications (IFITA), vol. 1, pp. 227-229, 2010.

[17] H. Chen, et al., "Research on a Heuristic Searching Algorithm for Energy-Aware Routing in Wireless Sensor Networks," Journal of Theoretical and Applied Information Technology, vol. 50, no.1, pp. 198-203, 2013.

[18] C. $\mathrm{Li}$, et al., "An energy-efficient unequal clustering mechanism for wireless sensor networks," in IEEE International Conference on Mobile Adhoc and Sensor Systems Conference, pp. 8-604, 2005.

[19] H. Bagci and A. Yazici, "An energy aware fuzzy unequal clustering algorithm for wireless sensor networks," in 2010 IEEE international conference on Fuzzy systems (FUZZ), pp. 1-8, 2010.

[20] S. Lee, et al., "Luca: An energy-efficient unequal clustering algorithm using location information for wireless sensor networks," Wireless Personal Communications, vol. 56, no. 4, pp. 715-731, 2011.

[21] C. J. Jiang, et al., "Energy-balanced unequal clustering protocol for wireless sensor networks," The Journal of China Universities of Posts and Telecommunications, vol. 17, no. 4, pp. 94-99, 2010.

[22] R. E. Korf, "Depth-first iterative-deepening: An optimal admissible tree search," Artificial Intelligence, vol. 27, no. 1, pp. 97-109, 1985.

[23] A. Mahanti, et al., "Performance of IDA* on trees and graphs," AAAI-92 Proceedings of the tenth national conference on artificial intelligene, pp. 539-544, 1992.

[24] V. N. Rao, et al., "Depth-first vs. best-first search," AAAI-91 Proceedings of the 9th National conference on artificial intelleigence, pp. 434-440, 1991.

[25] W. B. Heinzelman, et al., "An application-specific protocol architecture for wireless microsensor networks," IEEE Transactions on wireless communications, vol. 1, no. 4, pp. 660-670, 2002. 